\title{
Eine Formel für die Gesundheit
}

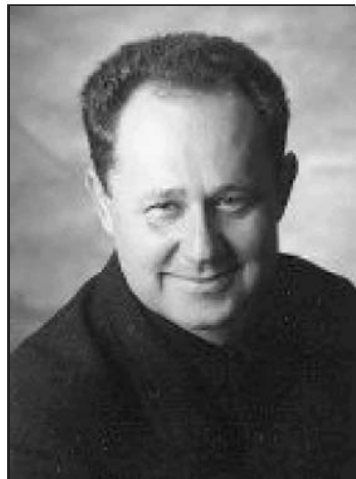

Prof. Dr. med. Manfred Wildner

Bibliografie

DOI http://dx.doi.org/

10.1055/s-0033-1363963

Gesundheitswesen 2014;

76: 5-6

(c) Georg Thieme Verlag KG Stuttgart · New York

ISSN 0941-3790

\section{Korrespondenzadresse}

Prof. Dr. med. Manfred Wildner

Bayerisches Landesamt für Gesundheit und Lebensmittelsicherheit

Veterinärstraße 2

85764 Oberschleißheim

manfred.wildner@|gl.bayern.de
Der Beginn eines neuen Jahres ist auch immer eine Zeit für neue Aufbrüche: Die Tage werden länger, und das zunehmende Licht leuchtet vielleicht auch in manchen Winkel privater Lebensführung. Und so wünschen wir uns selbst und anderen dann auch alles Gute, viel Glück und natürlich Gesundheit. Doch wie all dies erlangen? Und das auch noch auf eine Weise, auf welche sich die erhofften Güter nicht gegenseitig schaden? Hier wäre, zumindest für den Bereich Gesundheit, eine Gesundheitsformel höchst nützlich. An Vorschlägen dafür mangelt es nicht - das Spektrum umfasst die Fokussierung auf Einzelthemen wie Ernährungs- und Bewegungsregeln bis hin zu ganzheitlichen Ansätzen mit Bezug zu kosmischen Energien. Gewicht, Rauchen, Alkoholkonsum, Bewegung, Ernährung - diese im Akronym GRABE vereinten Risikofaktoren können uns buchstäblich zu „Grabe“ tragen. Oder uns eben, wenn sie als Gesundheitschancen verstanden werden, auch davor bewahren. Sie sind, im Gegensatz zu manch anderen Ansatzpunkten, mit solider Evidenz hinterlegt. Zumindest gilt das für ihre Eigenschaft als Gesundheitsdeterminanten. Wie sie in die gewünschte Richtung beeinflusst werden können, ist eine andere Frage.

Doch fehlt hier nicht etwas? Der Weg zur Hölle ist bekanntlich mit guten Vorsätzen gepflastert. Gute Absichten und gute Informationen zu gesundheitsförderlichem bzw. gesundheitsschädlichem Verhalten reichen für sich genommen häufig nicht für eine nachhaltige Verhaltensänderung aus. Zudem ist im Gesundheitsbereich mit wertenden Aussagen, wie sie mit "gut" und „böse“ impliziert sind, Vorsicht geboten. Die Bewertung von Gesundheit als erstrebens- und schützenswertes Gut, auch als zu schützendes und zu förderndes Recht, begründet damit noch keine Pflicht zur Gesundheit. Hier ist die menschliche Freiheit zu achten. Definiert man Gesundheit als die Voraussetzung für eine Persönlichkeitsentfaltung, welche sowohl eine bestmögliche individuelle Entwicklung wie auch eine produktive gesellschaftliche Teilhabe beinhaltet, wird schnell klar, dass es verschiedene individualisierte und personalisierte Formen von Gesundheit geben dürfte. Der Gesundheitsbegriff eines Pädagogen dürfte sich von dem einer Pianistin unterscheiden, der Gesundheitsbegriff eines Sportlehrers von dem eines Spitzenforschers. Aus den Problemen einer nachhaltigen gesundheitsförderlichen Verhaltensänderung wird deutlich, dass eine Formel für Gesundheit nicht die proximalen, personenzentrierten Faktoren wie das Gesundheitsverhalten alleine im Fokus ha- ben kann. Ein weiter gefasster Blick auf die Entstehung bzw. Beeinträchtigung von Gesundheit in der Wechselwirkung des Menschen mit seiner Umwelt ist unverzichtbar. Eine Übersicht über derartige Zusammenhänge gibt das Regenbogenmodell von Dahlgren und Whitehead, welches von den biologischen Faktoren über die Lebensstilfaktoren, die sozialen Netzwerke im näheren Lebensumfeld bis zu den breiteren kulturellen, sozioökonomischen und natürlichen Umweltfaktoren reicht [1].

Mit Abstrichen ließe sich damit in erster Näherung sagen, dass Gesundheit eine Funktion der ererbten biologischen persönlichen Voraussetzungen $(\mathrm{eB})$, des persönlichen Lebensstils $(\mathrm{pL})$ und der äußeren Umwelt $(\mathrm{eU})$ einschließlich der gesundheitlichen Versorgung ist, dies in direkten Ursache-Wirkungsbeziehungen oder auch vermittelt über Wechselwirkungen. Gesundheit $=\mathrm{f}$ (eB, pL, eU) (Detlef Ganten, bvoegd.de/rueckblick-2013)? Eine solche formelhafte Zusammenschau ist eine nützliche Merkhilfe, eine praktische Heuristik im Umgang mit gesundheitsrelevanten Themen. Sie hilft, wichtige Determinanten von Gesundheit und auch die Einflüsse ihrer Wechselwirkungen gegenwärtig zu halten. Eine Gesundheitsformel in einem strengeren Sinn ist dabei schon aufgrund definitorischer Unbestimmtheiten der Einflussfaktoren nicht so einfach zu haben. Zudem wäre auch noch eine empirische Überprüfung zu leisten. Ebenfalls im Blick zu behalten ist, dass menschliches Leben sich zwar auf physikochemische und biologische Prozesse gründet, durch diese jedoch nicht hinreichend und schon gar nicht deterministisch beschrieben ist. In einer SystemebenenBetrachtung wird deutlich, dass das menschliche Leben gegenüber tierischem und pflanzlichem Leben einzigartige und neue, emergente Eigenschaften aufweist, die annäherungsweise mit Begriffen wie Vernunft, Geist und Freiheit bezeichnet werden können. Damit ist zumindest eine biologische und geistige menschliche Doppelnatur festzustellen.

Eine Auswirkung dieser Neu-Emergenz des Geistes ist z. B., dass menschliches Handeln mehr durch Gründe bestimmt wird als durch Ursachen. Menschen handeln aus Gründen: Gründe, die in einer Reflexion vergangener Erfahrung liegen können oder aber in einer in die Zukunft gerichteten, aus ihr vorweggenommenen Hoffnung. Dass die subjektive Zukunftserwartung einen großen Einfluss auf gesundheitsrelevante Verhaltensweisen haben kann, ist beschrieben: Soziale Stabilität und positive Zukunftsaussichten sind 
mit gesundheitsorientiertem Handeln verbunden, gleichsam als Investition in eine positive Zukunft. Umgekehrt scheinen Unsicherheit und reduzierte Zukunftserwartungen mit riskanten Lebensweisen verknüpft.

In diesem Sinne ist der Mensch also nicht nur passiver Träger eines Gesundheitszustandes, sondern gleichzeitig auch handelndes Subjekt und damit Mitschöpfer bzw. Ko-Produzent von Gesundheit. Bezeichnenderweise wird selbst in den molekularbiologisch inspirierten Zukunftshoffnungen der P4-Medizin diese zentrale und proaktive, personale Rolle betont [2]. Diese entwirft das Zukunftsbild einer Konvergenz von Systembiologie, digitaler Revolution und verbraucherorientierter, gesundheitlicher Versorgung. Dabei steht eine aktive Teilhabe der häufig gesunden Nutzer an einer prädiktiven und in der Folge auch präventiven gesundheitlichen Versorgung im Zentrum. Die einzelne Person wird in Bezug gesetzt zu ihren digitalisierten Gesundheitsdaten. Von besonderer Bedeutung sind im P4-Medizinentwurf handlungsrelevante genetische Informationen, welche den Nutzer zur Stärkung seiner bzw. ihrer gesundheitsförderlichen Ressourcen einschließlich gesundheitsorientierten Verhaltens aktivieren sollen. Zielvorstellung: Der gesundheitsorientiert aktivierte und mit entsprechenden Ressourcen vernetzte kompetente Nutzer gesundheitlicher Angebote. Kritisch ließe sich anmerken, dass hier womöglich auch eine neue Art von Compliance im Sinne eines folgsamen Konsumenten angestrebt werden könnte.

Gleichzeitig ist eine Verantwortung des Menschen nicht nur für sich, sondern auch für seine Umwelt in all ihren Facetten anzusprechen. Die Verantwortung, alleine und in Gemeinschaft Bedingungen zu schaffen, in denen Menschen gesund sein können, wie es wegweisend formuliert worden ist [3]. Dieser kurze Satz enthält viel: Einerseits im Wort „Bedingungen“ die Einsicht, dass viele Elemente unserer Gesundheit nicht alleine verantwortet werden können, sondern von den äußeren Verhältnissen mit beeinflusst werden. Gleichzeitig die Zuversicht, dass sich diese Bedingungen gestalten lassen - ein Geschenk der Aufklärung und der Neuzeit. Darüber hinaus die Betonung der zentralen Rolle des Menschen bzw. von Menschengruppen im Zentrum und als Ziel dieser Bemühungen. Auch die Betonung der Gesundheit, also das ethische Primat der Prävention und Gesunderhaltung gegenüber einer reaktiven Behandlung von Krankheiten alleine. Und zuletzt findet sich im Wort „können“ die menschliche Freiheit versteckt: Eine Absage an alle Spielformen von „Gesundheitsdiktatur“, einschließlich eines Rechtes auf auch abweichendes Verhalten, soweit dies nicht die Rechte anderer Menschen beeinträchtigt: verantwortlicher Umgang, nicht Prohibition. Dies schließt die Verantwortung dafür ein, gesunde Entscheidungen leicht zu machen (make healthy choices easy choices) und sichere Umwelten zur Normalität. Das kann oft schon durch kleine Modifikationen erreicht werden: Gesundheit als zu berücksichtigendes Thema in allen Politik- und damit auch Lebensbereichen [4]. Und so wie ein afrikanisches Sprichwort sagt, dass ein ganzes Dorf benötigt wird, um ein Kind großzuziehen („it takes a village to raise a child“), kann vermutet werden, dass auch Gesundheit auf eine intaktes Netzwerk an persönlichen und gesellschaftlichen Ressourcen angewiesen ist: auf eine „gesunde Gesellschaft“ und damit auch auf ein „gesundes Gesundheitswesen“.

Mit den Fragestellungen einer gesunden Gesellschaft und eines gesunden Gesundheitswesens - hinsichtlich Risiken und hinsichtlich Ressourcen und Prozessen - beschäftigt sich auch diese Ausgabe unserer Zeitschrift: Zum Einfluss von Bildung und Schicht auf das Gesundheitsverhalten, zu hausärztlichem Nachwuchs und Karriereentscheidungen, zum Tabakkonsum im gesellschaftlichen Wandel, zu den Einflüssen auf die Krankheitskosten bei Systemischem Lupus Erythematodes, mit saisonaler Aktualität zu Influenza-Impfquoten und Einstellungen niedergelassener Ärzte sowie zur Influenza-Pandemie 2009/10 und dem Nutzen der Impfaktion in Hessen, zur externen Qualitätssicherung der Rehabilitationsangebote für Mütter/Väter und zum Zukunftsthema Medizintourismus und Medizinreisen. Wir freuen uns auch, im neuen Jahr wieder zertifizierte Beiträge zur ärztlichen Fortbildung (CME) anbieten zu können.

So könnte der Versuch einer Gesundheitsformel auf individuelle Ebene ergänzt werden durch den Versuch einer gesellschaftlichen Gesundheitsformel. Wie diese aussehen könnte? Vielleicht wäre sie von Hans Küngs Weltethos geprägt [5]: Durch eine Verpflichtung auf eine Kultur der Gewaltlosigkeit und der Ehrfurcht vor allem Leben, eine Verpflichtung auf eine Kultur der Solidarität und eine gerechte Wirtschaftsordnung, eine Verpflichtung auf eine Kultur der Toleranz und ein Leben in Wahrhaftigkeit, eine Verpflichtung auf eine Kultur der Gleichberechtigung und die Partnerschaft von Mann und Frau. In einer gesunden Gesellschaft wären Achtsamkeit und Respekt zwischen ihren Mitgliedern und gegenüber ihrer natürlichen und menschengemachten Umwelt unverzichtbar. Als „Formel“: Eine gesunde Gesellschaft $(g G)$ wäre damit gekennzeichnet durch das Bemühen um eine jeweils bessere Welt $(b W)$, in der Menschen füreinander da sind $(f M)$ und in einem wohlverstandenen Sinn aufeinander Acht haben $(a A)$. Im Erfoglsfall würde damit ein Gesellschaftsentwurf erfahrbar, der so attraktiv ist, dass er Menschen anzieht, um in ihm zu leben, zu lieben und zu arbeiten (Ottawa-Charta). In diesem Sinn uns allen ein gutes Neues Jahr!

\section{Literatur}

1 Dahlgren G, Whitehead M. Policies and Strategies to Promote Social Equity in Health. Stockholm: Institute for Futures Studies; 1991

2 P4 Medicine Institute. P4Medicine. URL http://p4mi.org/p4medicine Zugriff am 16.12.2013

3 Institute of Medicine. The Future of Public Health. 1988;URL http:// iom.edu/Reports/1988/The-Future-of-Public-Health.aspx Zugriff am 16.12.2013

4 Ståhl T, Wismar M, Ollila E et al. Health in all policies. 2006;URL http:// www.dors.it/alleg/newcms/201305/2006_HiAP_Filandia.pdf Zugriff am 16.12.2013

5 Parlament der Weltreligionen. Erklärung zum Weltethos vom 4.9.1993. URL http://www.weltethos.org/data-ge/c-10-stiftung/13-deklaration. php Zugriff am 16.12.2013 